\title{
Approximate controllability and optimal controls of fractional dynamical systems of order $1<q<2$ in Banach spaces
}

\author{
Haiyong Qin' ${ }^{1}$ Xin Zuo ${ }^{1 *}$, Jianwei Liu ${ }^{1}$ and Lishan Liu²
}

"Correspondence: zuox@cup.edu.cn ${ }^{1}$ Department of Automation, China University of Petroleum (Beijing), Beijing, 102249, China Full list of author information is available at the end of the article

\begin{abstract}
This paper investigates the approximate controllability and optimal controls of fractional dynamical systems of order $1<q<2$ in Banach spaces. We research a class of fractional dynamical systems governed by fractional integrodifferential equations with nonlocal initial conditions. Using the Krasnosel'skii fixed point theorem and the Schauder fixed point theorem, the approximate controllability results are obtained under two cases of the nonlinear term. We also present the existence results of optimal pairs of the corresponding fractional control systems with a Bolza cost function. Finally, an application is given to illustrate the effectiveness of our main results.
\end{abstract}

MSC: 26A33; 49J15; 49K27; 93B05; 93C25

Keywords: fractional integrodifferential equations; fixed point theorems; approximate controllability; optimal controls

\section{Introduction}

During the past two decades, fractional differential equations have been proved to be one of the most effective tools in the modeling of numerous fields of science, physics, engineering and so on. Since fractional differential equations efficiently describe many practical dynamical phenomena, they have attracted the attention of many researchers in the past years. Many authors investigated the existence of mild solutions of fractional differential equations by using semigroup theory and fixed point theorems (see [1-13]). Controllability is one of the most important issues in mathematical control theory and engineering, however, controllability of fractional dynamical systems is still in the initial stage [14-24]. Shu and Wang [7] considered the existence of mild solutions for a class of fractional integrodifferential equations of order $1<q<2$ in a Banach space:

$$
\left\{\begin{array}{l}
{ }^{C} D_{t}^{q} x(t)=A x(t)+f(t, x(t))+\int_{0}^{t} G(t-s) g(s, x(s)) d s, \quad 0<t<T, \\
x(0)+m(x)=x_{0} \in \mathbb{X}, \quad x^{\prime}(0)+n(x)=x_{1} \in \mathbb{X},
\end{array}\right.
$$

where ${ }^{C} D_{t}^{q}$ is Caputo's fractional derivative of order $1<q<2$. The existence results of mild solutions are obtained by the Krasnosel'skii fixed point theorem combined with solution operator theorem. Li et al. [15] studied controllability of the following differential systems

\section{Springer}

(C) 2015 Qin et al.; licensee Springer. This is an Open Access article distributed under the terms of the Creative Commons Attribution License (http://creativecommons.org/licenses/by/4.0), which permits unrestricted use, distribution, and reproduction in any medium, provided the original work is properly credited. 
of order $\alpha \in(1,2]$ with nonlocal conditions in an infinite dimensional Banach space:

$$
\left\{\begin{array}{l}
{ }^{C} D_{t}^{\alpha} x(t)=A x(t)+F(t, x(t))+B u(t), \quad t \in[0, b] \\
x(0)+g(x)=x_{0}, \quad x^{\prime}(0)=y_{0}
\end{array}\right.
$$

where $A$ is the infinitesimal generator of a strongly continuous $\alpha$-order cosine family $\left\{C_{\alpha}(t)\right\}_{t \geq 0}$ on a Banach space $\mathbb{X}$. The controllability results are obtained by using the Sadovskill fixed point theorem and vector-valued operator theory.

Recently, Sakthivel et al. [16] established the controllability results for a class of nonlinear fractional differential equations of order $1<q<2$ with nonlocal conditions. They also extended the main results to approximate controllability results for nonlocal fractional control systems with infinite delay. Wang et al. [25] obtained some existence and uniqueness results, and further existence conditions of optimal pairs for a class of fractional integrodifferential control systems were presented. Under the assumption that the associated linear system is approximately controllable, the approximate controllability of a class of semilinear fractional differential control systems are obtained in [17]. Since approximately controllable systems are more prevalent and practical than exact controllable ones, it is important to investigate the approximate controllability of semilinear differential systems that consists of a linear part and a nonlinear part.

However, to the best of our knowledge, most of the previous papers about fractional differential systems are concerned with the fractional derivative whose order is between zero and one, the approximate controllability problems for fractional integrodifferential equations in Caputo derivative sense of order $1<q<2$ have not been investigated extensively [25-29]. Especially, few researchers study the optimal control problems of fractional systems of order $1<q<2$ in Banach spaces. Liu et al. [27] investigated the existence and uniqueness of mild solutions and optimal controls for some fractional impulsive equations of order $1<q<2$. But the system under consideration does not include a Volterra operator in nonlinear term and the boundary conditions are local. What is more, the optimal control results are only applicable to Lagrange problems.

Motivated by [7, 15-17, 26, 27, 30-35], we discuss the approximate controllability and optimal controls of fractional dynamical systems of order $1<q<2$ in a Banach space. Consider the following fractional system:

$$
\left\{\begin{array}{l}
{ }^{C} D_{t}^{q} x(t)=A x(t)+f(t, x(t),(H x)(t))+B u(t), \quad t \in I=[0, b], \\
x(0)+g_{1}(x)=x_{0} \in \mathbb{X}, \quad x^{\prime}(0)+g_{2}(x)=x_{1} \in \mathbb{X}
\end{array}\right.
$$

where ${ }^{C} D_{t}^{q}$ is the Caputo fractional derivative of order $1<q<2$. $A: D(A) \subset \mathbb{X} \rightarrow \mathbb{X}$ is sectorial operator of type $(M, \theta, q, \mu)$ on a Banach space $\mathbb{X}$, endowed with the norm $\|\cdot\|$. $H: I \times I \times \mathbb{X} \rightarrow \mathbb{X}$ represents a Volterra-type operator, $(H x)(t)=\int_{0}^{t} h(t, s, x(s)) d s . B$ is a bounded linear operator from $U$ into $\mathbb{X}$, the control $u(\cdot)$ is given in $L^{2}(I, U), U$ is a Banach space. The nonlinear term $f: I \times \mathbb{X} \times \mathbb{X} \rightarrow \mathbb{X}$ is continuous, and nonlocal terms $g_{1}$ and $g_{2}$ are continuous functions.

The rest of the paper is organized as follows. In Section 2, we show some preliminaries and lemmas that are to be used later to prove our main results. In Section 3, we discuss approximate controllability of system (1.1). In Section 4, The existence of optimal controls of a class of semilinear fractional integrodifferential control systems are presented. Finally, an application is provided to illustrate the effectiveness of our main results in Section 5. 


\section{Preliminaries and lemmas}

Definition 2.1 (see [8]) The fractional integral of order $q$ with the lower limit zero for a function $f$ is defined as

$$
I^{q} f(t)=\frac{1}{\Gamma(q)} \int_{0}^{t} \frac{f(s)}{(t-s)^{1-q}} d s, \quad t>0, q>0
$$

provided that the right side is point-wise defined on $[0,+\infty)$, where $\Gamma(\cdot)$ is the gamma function.

Definition 2.2 (see [8]) The Riemann-Liouville derivative of the order $q$ with the lower limit zero for a function $f:[0, \infty] \rightarrow \mathbb{R}$ can be written as

$$
{ }^{L} D_{t}^{q} f(t)=\frac{1}{\Gamma(n-q)} \frac{d^{n}}{d t^{n}} \int_{0}^{t} \frac{f(s)}{(t-s)^{1-n+q}} d s, \quad t>0, n-1<q<n .
$$

Definition 2.3 (see [24]) The Caputo derivative of the order $q$ for a function $f:[0, \infty] \rightarrow$ $\mathbb{R}$ can be written as

$$
{ }^{C} D_{t}^{q} f(t)={ }^{L} D_{t}^{q}\left(f(t)-\sum_{k=0}^{n-1} \frac{t^{k}}{k !} f^{(k)}(0)\right), \quad t>0, n-1<q<n .
$$

\section{Remark 2.1}

(1) For $f:[0,+\infty) \rightarrow \mathbb{R}$

$$
{ }^{C} D_{t}^{q} f(t)=\frac{1}{\Gamma(n-q)} \int_{0}^{t} \frac{f^{(n)}(s)}{(t-s)^{1-n+q}} d s=I^{n-q} f^{(n)}(t), \quad t>0, n-1<q<n .
$$

(2) The Caputo derivative of a constant equals zero.

(3) If $f$ is an abstract function with values in $\mathbb{X}$, then the integrals which appear in Definitions 2.1, 2.2, and 2.3 are taken in Bochner's sense.

Definition 2.4 (see [7]) Let $A: D \subseteq \mathbb{X} \rightarrow \mathbb{X}$ be a closed and linear operator. A is said to be a sectorial operator of type $(M, \theta, q, \mu)$ if there exists $\mu \in \mathbb{R}, 0<\theta<\frac{\pi}{2}$, and $M>0$ such that the $q$-resolvent of $A$ exists outside the sector $\mu+S_{\theta}=\left\{\mu+\lambda^{q}: \lambda \in \mathbb{C},\left|\operatorname{Arg}\left(-\lambda^{q}\right)\right|<\theta\right\}$ and $\left\|R\left(\lambda^{q}, A\right)\right\| \leq \frac{M}{\left|\lambda^{q}-\mu\right|}, \lambda^{q} \notin \mu+S_{\theta}$.

Further, if $A$ is a sectorial operator of type $(M, \theta, q, \mu)$, then it is not difficult to see that $A$ is the infinitesimal generator of a $q$-resolvent family $\left\{\mathcal{T}_{q}(t)\right\}_{t \geq 0}$ in a Banach space, where $\mathcal{T}_{q}(t)=\frac{1}{2 \pi i} \int_{c} e^{\lambda t} R\left(\lambda^{q}, A\right) d \lambda$.

Lemma 2.1 (see [7]) Let $A$ be a sectorial operator of type $(M, \theta, q, \mu)$. If $f$ satisfies a uniform Hölder condition with exponent $\beta \in(0,1]$, the unique solution of linear fractional differential equation

$$
\left\{\begin{array}{l}
{ }^{C} D_{t}^{q} x(t)=A x(t)+f(t), \quad t \in I=[0, b], 1<q<2, \\
x(0)=x_{0} \in \mathbb{X}, \quad x^{\prime}(0)=x_{1} \in \mathbb{X}
\end{array}\right.
$$


is given by

$$
x(t)=\mathcal{S}_{q}(t) x_{0}+\mathcal{K}_{q}(t) x_{1}+\int_{0}^{t} \mathcal{T}_{q}(t-s) f(s) d s
$$

where

$$
\begin{aligned}
& \mathcal{S}_{q}(t)=\frac{1}{2 \pi i} \int_{c} e^{\lambda t} \lambda^{q-1} R\left(\lambda^{q}, A\right) d \lambda, \quad \mathcal{K}_{q}(t)=\frac{1}{2 \pi i} \int_{c} e^{\lambda t} \lambda^{q-2} R\left(\lambda^{q}, A\right) d \lambda, \\
& \mathcal{T}_{q}(t)=\frac{1}{2 \pi i} \int_{c} e^{\lambda t} R\left(\lambda^{q}, A\right) d \lambda,
\end{aligned}
$$

with $c$ being a suitable path such that $\lambda^{q} \notin \mu+S_{\theta}$ for $\lambda \in c$.

Definition 2.5 (see [16]) System (1.1) is said to be approximately controllable on $I$ if for every $x_{0}, x_{1} \in \mathbb{X}$, there is some control $u \in L^{2}(I, U)$, the closure of the reachable set $R(b)$ is dense in $\mathbb{X}$, i.e., $\overline{R(b)}=\mathbb{X}$, where $R(b)=\left\{x(b ; u): u(\cdot) \in L^{2}(I, U)\right\}$.

Consider the linear fractional control system

$$
\left\{\begin{array}{l}
{ }^{C} D_{t}^{q} x(t)=A x(t)+B u(t), \quad t \in[0, b], 1<q<2, \\
x(0)=x_{0} \in \mathbb{X}, \quad x^{\prime}(0)=x_{1} \in \mathbb{X} .
\end{array}\right.
$$

Let us now introduce the following operators. Define the operator $\Gamma_{0}^{b}: \mathbb{X} \rightarrow \mathbb{X}$ associated with $(2.8)$ as

$$
\begin{aligned}
& \Gamma_{0}^{b}=\int_{0}^{b} \mathcal{T}_{q}(b-s) B B^{*} \mathcal{T}_{q}^{*}(b-s) d s: \mathbb{X} \rightarrow \mathbb{X}, \\
& R\left(\lambda, \Gamma_{0}^{b}\right)=\left(\lambda I+\Gamma_{0}^{b}\right)^{-1}: \mathbb{X} \rightarrow \mathbb{X}, \quad \lambda>0,
\end{aligned}
$$

where $B^{*}$ denotes the adjoint of $B$ and $\mathcal{T}_{q}^{*}(t)$ is the adjoint of $\mathcal{T}_{q}(t)$. It is straightforward that the operator $\Gamma_{0}^{b}: \mathbb{X} \rightarrow \mathbb{X}$ is a linear bounded operator.

Lemma 2.2 (see [17]) The linear system (2.8) is approximately controllable if and only if $\lambda R\left(\lambda, \Gamma_{0}^{b}\right):=\lambda\left(\lambda I+\Gamma_{0}^{b}\right)^{-1} \rightarrow 0$ as $\lambda \rightarrow 0^{+}$in the strong operator topology.

In order to define the concept of mild solutions for problem (1.1), by the comparison with the fractional differential equation given in [1], we associate problem (1.1) to an integral problem.

Definition 2.6 A functional $x \in C(I, \mathbb{X})$ is called a mild solution for system (1.1) if for each $u \in L^{2}(I, U)$, the integral equation

$$
\begin{aligned}
x(t)= & \mathcal{S}_{q}(t)\left(x_{0}-g_{1}(x)\right)+\mathcal{K}_{q}(t)\left(x_{1}-g_{2}(x)\right) \\
& +\int_{0}^{t} \mathcal{T}_{q}(t-s)(f(s, x(s),(H x)(s))+B u(s)) d s
\end{aligned}
$$

is satisfied. 
Lemma 2.3 (Krasnosel'skii theorem; see [16]) Let $\mathbb{X}$ be a Banach space and $E$ be a bounded, closed, and convex subset of $\mathbb{X}$. Let $Q_{1}, Q_{2}$ be maps of $E$ into $\mathbb{X}$ such that $Q_{1} x+Q_{2} y \in E$, for every $x, y \in E$. If $Q_{1}$ is contraction and $Q_{2}$ is compact and continuous, then the equation $Q_{1} x+Q_{2} x=x$ has a solution on $E$.

Lemma 2.4 (Sadovskill theorem; see [15]) Let $Q$ be a condensing operator on a Banach space $\mathbb{X}$, i.e. $Q$ is continuous and takes bounded sets into bounded sets, and $\partial(Q(D))<\partial(D)$ for every bounded set $D$ of $\mathbb{X}$ with $\partial(D)>0$. If $Q(E) \subset E$ for a convex, closed, and bounded set $E$ of $\mathbb{X}$, then $Q$ has a fixed point in $E$, where $\partial(\cdot)$ denotes the Kuratowski measure of noncompactness.

\section{Approximate controllability}

From Theorems 3.3 and 3.4 in [7], it is easy to see that $\mathcal{S}_{q}(t), \mathcal{K}_{q}(t)$, and $\mathcal{T}_{q}(t)$ are bounded. Define $k^{*}=\sup _{t \in I} \int_{0}^{b} m(t, s) d s<\infty$. For any $r>0, B_{r}:=\{x \in C(I, \mathbb{X}) \mid\|x\| \leq r\}, C(I, \mathbb{X}) \mathrm{de}-$ notes a Banach space with the norm $\|x\|=\sup _{t \in I}\|x(t)\| .\|B\| \leq M_{B}$. Here we impose the following assumptions:

$\left(\mathrm{H}_{1}\right)$ The operators $\mathcal{S}_{q}(t), \mathcal{K}_{q}(t), \mathcal{T}_{q}(t)$ generated by $A$ are compact in $\overline{D(A)}$ when $t \geq 0$ such that

$$
\sup _{t \in I}\left\|\mathcal{S}_{q}(t)\right\| \leq M, \quad \sup _{t \in I}\left\|\mathcal{K}_{q}(t)\right\| \leq M, \quad \sup _{t \in I}\left\|\mathcal{T}_{q}(t)\right\| \leq M
$$

$\left(\mathrm{H}_{2}\right)$ The nonlinearity $f: I \times \mathbb{X} \times \mathbb{X} \rightarrow \mathbb{X}$ is continuous, there exist positive functions $\mu_{i} \in$ $L^{\infty}\left(I, \mathbb{R}^{+}\right)(i=1,2,3)$ such that

$$
\|f(t, x, y)\| \leq \mu_{1}(t)+\mu_{2}(t)\|x\|+\mu_{3}(t)\|y\| .
$$

$\left(\mathrm{H}_{2}^{\prime}\right)$ The nonlinearity $f: I \times \mathbb{X} \times \mathbb{X} \rightarrow \mathbb{X}$ is continuous and compact, there exist positive constants $\alpha_{1}, \alpha_{2}$ such that

$$
\|f(t, x, H x)-f(t, y, H y)\| \leq \alpha_{1}\|x-y\|+\alpha_{2}\|H x-H y\| .
$$

$\left(\mathrm{H}_{3}\right)$ The functions $g_{1}, g_{2}: \mathbb{X} \rightarrow \overline{D(A)}$ are completely continuous and there exist positive constants $\beta_{1}, \beta_{2}$ such that

$$
\left\|g_{1}(x)-g_{1}(y)\right\| \leq \beta_{1}\|x-y\|, \quad\left\|g_{2}(x)-g_{2}(y)\right\| \leq \beta_{2}\|x-y\|, \quad x, y \in \mathbb{X} .
$$

$\left(\mathrm{H}_{4}\right) h: \Delta \times \mathbb{X} \rightarrow \mathbb{X}$, there exists a function $m(t, s) \in C\left(\Delta, \mathbb{R}^{+}\right)$such that

$$
\|h(t, s, x(s))\| \leq m(t, s)\|x\|
$$

for each $(t, s) \in \Delta$ and $x, y \in \mathbb{X}$, where $\Delta=\left\{(t, s) \in \mathbb{R}^{2} \mid 0 \leq s, t \leq b\right\}$.

Theorem 3.1 Assume that conditions $\left(\mathrm{H}_{1}\right),\left(\mathrm{H}_{2}\right)$, and $\left(\mathrm{H}_{3}\right)-\left(\mathrm{H}_{4}\right)$ hold and, in addition, the functions $f(t, x, H x)$ and $h(t, s, x)$ are bounded for $t \in[0, b], x \in \mathbb{X}$. The linear system (2.8) is approximately controllable. Then the fractional control system (1.1) is approximately controllable on $[0, b]$ provided that $M\left(\beta_{1}+\beta_{2}\right)<1$. 
Proof Define the operators $Q_{1}$ and $Q_{2}$ on $B_{r}$ as follows:

$$
\begin{aligned}
& \left(Q_{1} x\right)(t)=\mathcal{S}_{q}(t)\left(x_{0}-g_{1}(x)\right)+\mathcal{K}_{q}(t)\left(x_{1}-g_{2}(x)\right) \\
& \left(Q_{2} x\right)(t)=\int_{0}^{t} \mathcal{T}_{q}(t-s) B u(s) d s+\int_{0}^{t} \mathcal{T}_{q}(t-s) f(s, x(s),(H x)(s)) d s
\end{aligned}
$$

From [16], system (1.1) is approximately controllable, if for any $\lambda>0$, there exists a continuous function $x(\cdot) \in C(I, \mathbb{X})$ such that

$$
\begin{aligned}
x(t)= & \mathcal{S}_{q}(t)\left(x_{0}-g_{1}(x)\right)+\mathcal{K}_{q}(t)\left(x_{1}-g_{2}(x)\right)+\int_{0}^{t} \mathcal{T}_{q}(t-s) B u(s) d s \\
& +\int_{0}^{t} \mathcal{T}_{q}(t-s) f(s, x(s),(H x)(s)) d s, \\
u(t)= & B^{*} \mathcal{T}_{q}^{*}(b-t) R\left(\lambda, \Gamma_{0}^{b}\right) p(x(\cdot)),
\end{aligned}
$$

where

$$
\begin{aligned}
p(x(\cdot))= & x_{b}-\mathcal{S}_{q}(b)\left(x_{0}-g_{1}(x)\right)-\mathcal{K}_{q}(b)\left(x_{1}-g_{2}(x)\right) \\
& -\int_{0}^{b} \mathcal{T}_{q}(b-s) f(s, x(s),(H x)(s)) d s .
\end{aligned}
$$

For any $\lambda>0$, we choose $r>\left(1+\frac{1}{\lambda} M_{B}^{2} M^{2} b\right)\left(C_{1}+C_{2}\right)$. Next, we shall show that $Q_{1}+Q_{2}$ has a fixed point on $B_{r}$, which is then a solution of system (1.1). In view of assumptions $\left(\mathrm{H}_{1}\right),\left(\mathrm{H}_{2}\right)$, and $\left(\mathrm{H}_{3}\right)-\left(\mathrm{H}_{4}\right)$, we have

$$
\begin{aligned}
\|u(t)\| \leq & \frac{1}{\lambda} M_{B} M\left(\left\|x_{b}\right\|+\left\|\mathcal{S}_{q}(b)\right\|\left\|x_{0}-g_{1}(x)\right\|+\left\|\mathcal{K}_{q}(b)\right\|\left\|x_{1}-g_{2}(x)\right\|\right. \\
& \left.+\int_{0}^{b}\left\|\mathcal{T}_{q}(b-s)\right\|\|f(s, x(s),(H x)(s))\| d s\right) \\
\leq & \frac{1}{\lambda} M_{B} M\left[\left\|x_{b}\right\|+M\left(\left\|x_{0}\right\|+\left\|g_{1}(x)\right\|+\left\|x_{1}\right\|+\left\|g_{2}(x)\right\|\right)\right. \\
& \left.+M b\left(\left\|\mu_{1}\right\|_{L^{\infty}\left[I, \mathbb{R}^{+}\right]}+r\left\|\mu_{2}\right\|_{L^{\infty}\left[I, \mathbb{R}^{+}\right]}+k^{*} r\left\|\mu_{3}\right\|_{L^{\infty}\left[I, \mathbb{R}^{+}\right]}\right)\right] \\
\leq & \frac{1}{\lambda} M_{B} M\left(C_{1}+C_{2}\right),
\end{aligned}
$$

where $C_{1}=\left\|x_{b}\right\|+M\left\|x_{0}\right\|+M \beta_{1} r+M g_{1}(0)+M\left\|x_{1}\right\|+M \beta_{2} r+M g_{2}(0), C_{2}=M b\left(\left\|\mu_{1}\right\|_{L^{\infty}\left[I, \mathbb{R}^{+}\right]}+\right.$ $\left.r\left\|\mu_{2}\right\|_{L^{\infty}\left[I, \mathbb{R}^{+}\right]}+k^{*} r\left\|\mu_{3}\right\|_{L^{\infty}\left[I, \mathbb{R}^{+}\right]}\right)$.

For any $x \in B_{r}$, we obtain

$$
\begin{aligned}
\left\|\left(Q_{1} x\right)(t)+\left(Q_{2} x\right)(t)\right\| \leq & M\left\|x_{0}\right\|+M\left\|g_{1}(x)\right\|+M\left\|x_{1}\right\|+M\left\|g_{2}(x)\right\|+M M_{B} b\|u(t)\| \\
& +M b\left(\left\|\mu_{1}\right\|_{L^{\infty}\left[I, \mathbb{R}^{+}\right]}+r\left\|\mu_{2}\right\|_{L^{\infty}\left[I, \mathbb{R}^{+}\right]}+k^{*} r\left\|\mu_{3}\right\|_{L^{\infty}\left[I, \mathbb{R}^{+}\right]}\right) \\
\leq & \left(1+\frac{1}{\lambda} M_{B}^{2} M^{2} b\right)\left(C_{1}+C_{2}\right)
\end{aligned}
$$

Hence, we conclude that $\left\|\left(Q_{1} x\right)(t)+\left(Q_{2} x\right)(t)\right\| \leq r$. 
Using assumptions $\left(\mathrm{H}_{1}\right)$ and $\left(\mathrm{H}_{3}\right)$, for any $x, y \in B_{r}$ and $t \in[0, b]$, we have

$$
\begin{aligned}
\left\|\left(Q_{1} x\right)(t)-\left(Q_{1} y\right)(t)\right\| & \leq\left\|\mathcal{S}_{q}(t)\right\|\left\|g_{1}(x)-g_{1}(y)\right\|+\left\|\mathcal{K}_{q}(t)\right\|\left\|g_{2}(x)-g_{2}(y)\right\| \\
& \leq M\left(\beta_{1}+\beta_{2}\right)\|x-y\| .
\end{aligned}
$$

Since $M\left(\beta_{1}+\beta_{2}\right)<1$, it follows that $Q_{1}$ is a contraction mapping.

Let $\left\{x_{n}\right\}$ be a sequence in $B_{r}$, and $x_{n} \rightarrow x \in B_{r}$. Because $f, g_{1}$, and $g_{2}$ are continuous, i.e., for all $\varepsilon>0$, there exists a positive integer $N$, when $n>N$, we obtain

$$
\begin{aligned}
& \left\|f\left(s, x_{n}(s),\left(H x_{n}\right)(s)\right)-f(s, x(s),(H x)(s))\right\| \leq \varepsilon, \quad\left\|g_{1}\left(x_{n}\right)-g_{1}(x)\right\| \leq \varepsilon, \\
& \left\|g_{2}\left(x_{n}\right)-g_{2}(x)\right\| \leq \varepsilon
\end{aligned}
$$

Now, for all $t \in[0, b]$, we infer that

$$
\begin{aligned}
& \left\|\left(Q_{2} x_{n}\right)(t)-\left(Q_{2} x\right)(t)\right\| \\
& \leq \int_{0}^{t}\left\|\mathcal{T}_{q}(t-\tau)\right\|\left\|B B^{*} \mathcal{T}_{q}^{*}(b-\tau) R\left(\lambda, \Gamma_{0}^{b}\right)\right\| \\
& \quad \times\left(\left\|\mathcal{S}_{q}(b)\left(g_{1}\left(x_{n}\right)-g_{1}(x)\right)\right\|+\left\|\mathcal{K}_{q}(b)\left(g_{2}\left(x_{n}\right)-g_{2}(x)\right)\right\|\right. \\
& \left.\quad+\int_{0}^{b}\left\|\mathcal{T}_{q}(b-s)\right\|\left\|f\left(s, x_{n}(s),\left(H x_{n}\right)(s)\right)-f(s, x(s),(H x)(s))\right\| d s\right) d \tau \\
& \quad+\int_{0}^{t} \mathcal{T}_{q}(t-s)\left(f\left(s, x_{n},\left(H x_{n}\right)(s)\right)-f(s, x(s),(H x)(s))\right) d s \\
& \leq\left(\frac{1}{\lambda} M_{B}^{2} M^{2} b(2 M+M b)+M b\right) \varepsilon
\end{aligned}
$$

This implies that $Q_{2}$ is continuous.

Now, we prove that compactness of $Q_{2}$. To prove this, we first prove that the set $\left\{\left(Q_{2} x\right)(t): x \in B_{r}\right\}$ is relatively compact in $C(I, \mathbb{X})$.

By the assumptions of this theorem, it is easy to see that

$$
\left\|\left(Q_{2} x\right)(t)\right\| \leq M M_{B} b+M b\left(\left\|\mu_{1}\right\|_{L^{\infty}\left[I, \mathbb{R}^{+}\right]}+r\left\|\mu_{2}\right\|_{L^{\infty}\left[I, \mathbb{R}^{+}\right]}+k^{*} r\left\|\mu_{3}\right\|_{L^{\infty}\left[I, \mathbb{R}^{+}\right]}\right),
$$

so we know that $\left\{\left(Q_{2} x\right)(t): x \in B_{r}\right\}$ is uniformly bounded. Then we show that $Q_{2}\left(B_{r}\right)$ is equicontinuous. The functions $\left\{\left(Q_{2} x\right)(t): x \in B_{r}\right\}$ are equicontinuous at $t=0$. For any $x \in$ $B_{r}$ and $0<t_{1}<t_{2} \leq b$, we have

$$
\begin{aligned}
& \left\|\left(Q_{2} x\right)\left(t_{2}\right)-\left(Q_{2} x\right)\left(t_{1}\right)\right\| \\
& \leq\left\|\int_{0}^{t_{1}}\left[\mathcal{T}_{q}\left(t_{2}-s\right)-\mathcal{T}_{q}\left(t_{1}-s\right)\right] B u(s) d s\right\|+\left\|\int_{t_{1}}^{t_{2}} \mathcal{T}_{q}\left(t_{2}-s\right) B u(s) d s\right\| \\
& \quad+\left\|\int_{0}^{t_{1}}\left[\mathcal{T}_{q}\left(t_{2}-s\right)-\mathcal{T}_{q}\left(t_{1}-s\right)\right] f(s, x(s),(H x)(s)) d s\right\| \\
& \quad+\left\|\int_{t_{1}}^{t_{2}} \mathcal{T}_{q}\left(t_{2}-s\right) f(s, x(s),(H x)(s)) d s\right\|
\end{aligned}
$$




$$
\begin{aligned}
\leq & M_{B} \int_{0}^{t_{1}}\left\|\mathcal{T}_{q}\left(t_{2}-s\right)-\mathcal{T}_{q}\left(t_{1}-s\right)\right\| d s\|u\|+M M_{B}\left(t_{2}-t_{1}\right)\|u\| \\
& +M\left(t_{2}-t_{1}\right)\|f(s, x(s),(H x)(s))\|+\int_{0}^{t_{1}}\left\|\mathcal{T}_{q}\left(t_{2}-s\right)-\mathcal{T}_{q}\left(t_{1}-s\right)\right\| d s \\
& \times\left(\left\|\mu_{1}\right\|_{L^{\infty}\left[I, \mathbb{R}^{+}\right]}+r\left\|\mu_{2}\right\|_{L^{\infty}\left[I, \mathbb{R}^{+}\right]}+k^{*} r\left\|\mu_{3}\right\|_{L^{\infty}\left[I, \mathbb{R}^{+}\right]}\right) .
\end{aligned}
$$

By the continuity of the function $t \rightarrow\left\|\mathcal{T}_{q}(t)\right\|$, the right hand side of the above inequality tends to zero as $t_{2} \rightarrow t_{1}$. Therefore, $\left\{\left(Q_{2} x\right)(t): x \in B_{r}\right\}$ is a family of equicontinuous functions.

According to the infinite dimensional version of the Ascoli-Arzela theorem, it remains to prove that for any $t \in[0, b]$, the set $V(t):=\left\{\left(Q_{2} x\right)(t): x \in B_{r}\right\}$ is relatively compact in $C(I, \mathbb{X})$. The case $t=0$ is trivial, $V(0)=\left\{\left(Q_{2} x\right)(0): x(\cdot) \in B_{r}\right\}$ is compact in $C(I, \mathbb{X})$. Let $t \in(0, b]$ be a fixed real number, and let $h$ be a given real number satisfied $0<h<t$, define $V_{h}(t)=\left\{\left(Q_{2}^{h} x\right)(t): x \in B_{r}\right\}$,

$$
\begin{aligned}
\left(Q_{2}^{h} x\right)(t)= & \int_{0}^{t-h} \mathcal{T}_{q}(t-s) B u(s) d s+\int_{0}^{t-h} \mathcal{T}_{q}(t-s) f(s, x(s),(H x)(s)) d s \\
= & \mathcal{T}_{q}(h) \int_{0}^{t-h} \mathcal{T}_{q}(t-s-h) B u(s) d s \\
& +\mathcal{T}_{q}(h) \int_{0}^{t-h} \mathcal{T}_{q}(t-s-h) f(s, x(s),(H x)(s)) d s \\
= & \mathcal{T}_{q}(h) y(t, h) .
\end{aligned}
$$

Since $\mathcal{T}_{q}(h)$ is compact in $C(I, \mathbb{X})$ and $y(t, h)$ is bounded on $B_{r}$, then the set $V_{h}(t)$ is relatively compact in $C(I, \mathbb{X})$. Since

$$
\begin{aligned}
& \left\|\left(Q_{2} x\right)(t)-\left(Q_{2}^{h} x\right)(t)\right\| \\
& \leq \int_{t-h}^{t} \mathcal{T}_{q}(t-s) B u(s) d s+\int_{t-h}^{t} \mathcal{T}_{q}(t-s) f(s, x(s), H x(s)) d s \\
& \leq \frac{1}{\lambda} M^{2} M_{B}^{2}\left(C_{1}+C_{2}\right) \int_{t-h}^{t} d s \\
& \quad+M\left(\left\|\mu_{1}\right\|_{L^{\infty}\left[I, \mathbb{R}^{+}\right]}+r\left\|\mu_{2}\right\|_{L^{\infty}\left[I, \mathbb{R}^{+}\right]}+k^{*} r\left\|\mu_{3}\right\|_{L^{\infty}\left[I, \mathbb{R}^{+}\right]}\right) \int_{t-h}^{t} d s,
\end{aligned}
$$

if $h$ is small enough, it implies that there are relatively compact sets arbitrarily close to the set $V(t)$ for each $t \in(0, b]$. Then $V(t), t \in(0, b]$ is relatively compact in $C(I, \mathbb{X})$. Since it is compact at $t=0$, we have the relatively compactness of $V(t)$ in $C(I, \mathbb{X})$ for all $t \in[0, b]$. Hence, by the Arzela-Ascoli theorem, we obtain the result that $Q_{2}$ is compact. In view of Lemma 2.3, we can conclude that the control system (1.1) has at least one mild solution on $[0, b]$.

Without loss of generality, we assume that $x_{\lambda}(\cdot)$ is a fixed point of $Q_{1}+Q_{2}$ in $B_{r}$. Then from [16], any fixed point of $Q_{1}+Q_{2}$ is a mild solution of $(1.1)$ on $[0, b]$ under the control

$$
u_{\lambda}(t)=B^{*} \mathcal{T}_{q}^{*}(b-t) R\left(\lambda, \Gamma_{0}^{b}\right) p\left(x_{\lambda}\right), \quad t \in I,
$$

and satisfies $x_{\lambda}(b)=x_{b}-\lambda R\left(\lambda, \Gamma_{0}^{b}\right) p\left(x_{\lambda}\right)$. 
The functions $f(t, x, H x)$ and $h(t, s, x)$ are bounded for $t \in[0, b]$ and $x \in \mathbb{X}$, thus there exists a constant $N>0$ such that

$$
\int_{0}^{b}\left\|f\left(s, x_{\lambda}(s),\left(H x_{\lambda}\right)(s)\right)\right\|^{2} d s \leq b N^{2}
$$

Consequently, there is a sequence, still denoted by $\left\{f\left(s, x_{\lambda}(s),\left(H x_{\lambda}\right)(s)\right)\right\}$, that weakly converges to say $\{f(s)\}$ in $L^{2}[I, \mathbb{X}]$. Denote

$$
w=x_{b}-\mathcal{S}_{q}(b)\left(x_{0}-g_{1}\left(x_{\lambda}\right)\right)-\mathcal{K}_{q}(b)\left(x_{1}-g_{2}\left(x_{\lambda}\right)\right)-\int_{0}^{b} \mathcal{T}_{q}(b-s) f(s) d s
$$

From (3.4) and (3.16), we know that

$$
\begin{aligned}
\left\|p\left(x_{\lambda}\right)-w\right\| & =\left\|\int_{0}^{b} \mathcal{T}_{q}(b-s)\left[f\left(s, x_{\lambda},\left(H x_{\lambda}\right)(s)\right)-f(s)\right] d s\right\| \\
& \leq \sup _{t \in[0, b]}\left\|\int_{0}^{t} \mathcal{T}_{q}(t-s)\left[f\left(s, x_{\lambda},\left(H x_{\lambda}\right)(s)\right)-f(s)\right] d s\right\| .
\end{aligned}
$$

By using the infinite dimensional version of the Ascoli-Arzela theorem, one can show that the operator $l(\cdot) \rightarrow \int_{0}^{\cdot} \mathcal{T}_{q}(\cdot-s) l(s) d s: L^{2}[I, \mathbb{X}] \rightarrow C(I, \mathbb{X})$ is compact. Therefore, for all $t \in$ $[0, b]$, we obtain $\left\|p\left(x_{\lambda}\right)-w\right\| \rightarrow 0$ as $\lambda \rightarrow 0^{+}$.

$$
\begin{aligned}
\left\|x_{\lambda}(b)-x_{b}\right\| & \leq\left\|\lambda R\left(\lambda, \Gamma_{0}^{b}\right)(w)\right\|+\left\|\lambda R\left(\lambda, \Gamma_{0}^{b}\right)\right\|\left\|p\left(x_{\lambda}\right)-w\right\| \\
& \leq\left\|\lambda R\left(\lambda, \Gamma_{0}^{b}\right)(w)\right\|+\left\|p\left(x_{\lambda}\right)-w\right\| .
\end{aligned}
$$

It follows that $\left\|x_{\lambda}(b)-x_{b}\right\| \rightarrow 0$ as $\lambda \rightarrow 0^{+}$. From Lemma 2.2 , we know that the fractional control system (1.1) is approximately controllable on $[0, b]$. The proof is completed.

Theorem 3.2 Assume that conditions $\left(\mathrm{H}_{1}\right),\left(\mathrm{H}_{2}^{\prime}\right)$, and $\left(\mathrm{H}_{3}\right)-\left(\mathrm{H}_{4}\right)$ hold. The functions $f(t, x, H x)$ and $h(t, s, x)$ are bounded for $t \in[0, b], x \in \mathbb{X}$. The linear system (2.8) is approximately controllable. Then the fractional control system (1.1) is approximately controllable on $[0, b]$ provided that $\left(1+\frac{1}{\lambda} M_{B}^{2} M^{2} b\right)\left(M \beta_{1}+M \beta_{2}+\alpha_{1} b M+\alpha_{2} b k^{*} M\right)<1$.

Proof Define the operators $Q_{1}^{\prime}$ and $Q_{2}^{\prime}$ on $B_{r}$ as follows:

$$
\begin{aligned}
& \left(Q_{1}^{\prime} x\right)(t)=\mathcal{S}_{q}(t)\left(x_{0}-g_{1}(x)\right)+\mathcal{K}_{q}(t)\left(x_{1}-g_{2}(x)\right)+\int_{0}^{t} \mathcal{T}_{q}(t-s) B u(s) d s \\
& \left(Q_{2}^{\prime} x\right)(t)=\int_{0}^{t} \mathcal{T}_{q}(t-s) f(s, x(s),(H x)(s)) d s
\end{aligned}
$$

By the definition, it is easy to see that $B_{r}$ is a bounded, closed, and convex set in $C(I, \mathbb{X})$. We shall prove that there exists a constant $r>0$ such that $\left(Q_{1}^{\prime}+Q_{2}^{\prime}\right)\left(B_{r}\right) \subset B_{r}$. If this is not true, then for each $r>0$, there exists $x_{\lambda} \in B_{r}$, but $\left(Q_{1}^{\prime}+Q_{2}^{\prime}\right)\left(B_{r}\right)$ does not belong to $B_{r}$, i.e., $\left\|\left(Q_{1}^{\prime}+Q_{2}^{\prime}\right)\left(x_{\lambda}\right)(t)\right\|>r$ for some $t \in[0, b]$. 
In view of assumptions $\left(\mathrm{H}_{1}\right),\left(\mathrm{H}_{2}^{\prime}\right)$, and $\left(\mathrm{H}_{3}\right)-\left(\mathrm{H}_{4}\right)$, we have

$$
\begin{aligned}
\|u(t)\| \leq & \frac{1}{\lambda} M_{B} M\left(\left\|x_{b}\right\|+M\left(\left\|x_{0}\right\|+\left\|g_{1}\left(x_{\lambda}\right)\right\|+\left\|x_{1}\right\|+\left\|g_{2}\left(x_{\lambda}\right)\right\|\right)\right. \\
& \left.+M \int_{0}^{b}\left\|f\left(s, x_{\lambda}(s),\left(H x_{\lambda}\right)(s)\right)\right\| d s\right) \\
\leq & \frac{1}{\lambda} M_{B} M\left(\left\|x_{b}\right\|+M\left(\left\|x_{0}\right\|+\beta_{1}\left\|x_{\lambda}\right\|+g_{1}(0)+\left\|x_{1}\right\|+\beta_{2}\left\|x_{\lambda}\right\|+g_{2}(0)\right)\right. \\
& \left.+M \int_{0}^{b}\left\|f\left(s, x_{\lambda}(s),\left(H x_{\lambda}\right)(s)\right)\right\| d s\right) \\
\leq & \frac{1}{\lambda} M_{B} M\left(\left\|x_{b}\right\|+M\left(\left\|x_{0}\right\|+\beta_{1}\left\|x_{\lambda}\right\|+g_{1}(0)+\left\|x_{1}\right\|+\beta_{2}\left\|x_{\lambda}\right\|+g_{2}(0)\right)\right. \\
& \left.+\alpha_{1} b M\left\|x_{\lambda}\right\|+\alpha_{2} b k^{*} M\left\|x_{\lambda}\right\|+M \int_{0}^{b}\|f(s, 0,0)\| d s\right) .
\end{aligned}
$$

From (3.19), we get

$$
\begin{gathered}
\left\|\left(Q_{1}^{\prime} x_{\lambda}\right)(t)\right\| \leq M\left\|x_{0}\right\|+M\left\|g_{1}\left(x_{\lambda}\right)\right\|+M\left\|x_{1}\right\|+M\left\|g_{2}\left(x_{\lambda}\right)\right\|+M \int_{0}^{b}\|B u(s)\| d s \\
\leq M\left(\left\|x_{0}\right\|+\beta_{1}\left\|x_{\lambda}\right\|+g_{1}(0)+\left\|x_{1}\right\|+\beta_{2}\left\|x_{\lambda}\right\|+g_{2}(0)\right) \\
+M M_{B} \int_{0}^{b}\|u(s)\| d s \\
\left(Q_{2}^{\prime} x_{\lambda}\right)(t) \leq \alpha_{1} b M\left\|x_{\lambda}\right\|+\alpha_{2} b k^{*} M\left\|x_{\lambda}\right\|+M \int_{0}^{b}\|f(s, 0,0)\| d s .
\end{gathered}
$$

From (3.21)-(3.22) and $x_{\lambda} \leq r$, it follows that

$$
\begin{aligned}
& r<\left\|\left(Q_{1}^{\prime}+Q_{2}^{\prime}\right) x_{\lambda}(t)\right\| \\
& \leq M\left(\left\|x_{0}\right\|+\beta_{1}\left\|x_{\lambda}\right\|+g_{1}(0)+\left\|x_{1}\right\|+\beta_{2}\left\|x_{\lambda}\right\|+g_{2}(0)\right)+M M_{B} \int_{0}^{b}\|u(s)\| d s \\
& \quad+\alpha_{1} b M\left\|x_{\lambda}\right\|+\alpha_{2} b k^{*} M\left\|x_{\lambda}\right\|+M \int_{0}^{b}\|f(s, 0,0)\| d s .
\end{aligned}
$$

Dividing both sides of (3.23) by $r$ and taking the limit as $r \rightarrow \infty$, we obtain

$$
\left(1+\frac{1}{\lambda} M_{B}^{2} M^{2} b\right)\left(M \beta_{1}+M \beta_{2}+\alpha_{1} b M+\alpha_{2} b k^{*} M\right) \geq 1
$$

This contradicts the assumption in this theorem. Thus we can deduce that $\left(Q_{1}^{\prime}+Q_{2}^{\prime}\right)\left(B_{r}\right) \subset$ $B_{r}$.

Next, we show that $Q_{1}^{\prime}$ is a contraction operator, and $Q_{2}^{\prime}$ is a completely continuous operator. Assume that $x, y \in B_{r}$, we conclude that

$$
\begin{aligned}
& \left\|\left(Q_{1}^{\prime} x\right)(t)-\left(Q_{1}^{\prime} y\right)(t)\right\| \\
& \quad \leq M \beta_{1}\|x-y\|+M \beta_{2}\|x-y\|
\end{aligned}
$$




$$
\begin{aligned}
& +\frac{1}{\lambda} M^{3} M_{B}^{2} b\left(\beta_{1}\|x-y\|+\beta_{2}\|x-y\|+\alpha_{1} b\|x-y\|+\alpha_{2} b k^{*}\|x-y\|\right) \\
\leq & \left(\left(M \beta_{1}+M \beta_{2}\right)+\frac{1}{\lambda} M^{2} M_{B}^{2} b\left(M \beta_{1}+M \beta_{2}+\alpha_{1} M b+\alpha_{2} M b k^{*}\right)\right)\|x-y\| \\
\leq & \left(1+\frac{1}{\lambda} M_{B}^{2} M^{2} b\right)\left(M \beta_{1}+M \beta_{2}+\alpha_{1} M b+\alpha_{2} M b k^{*}\right)\|x-y\| .
\end{aligned}
$$

By the assumptions in this theorem, we know that $Q_{1}^{\prime}$ is a contraction operator.

Let $x_{n} \in B_{r}$ with $x_{n} \rightarrow x$ in $B_{r}$. By $\left(\mathrm{H}_{2}^{\prime}\right)$, it follows that

$$
f\left(s, x_{n}(s),\left(H x_{n}\right)(s)\right) \rightarrow f(s, x(s),(H x)(s)), \quad n \rightarrow \infty
$$

and

$$
\left\|f\left(s, x_{n}(s),\left(H x_{n}\right)(s)\right)-f(s, x(s),(H x)(s))\right\| \leq \alpha_{1}\left\|x_{n}-x\right\|+\alpha_{2}\left\|H x_{n}-H x\right\| .
$$

From (3.27) and the dominated convergence theorem, it is easy to see that $Q_{2}^{\prime}$ is continuous on $B_{r}$.

For any $x \in B_{r}$ and $h>0$, we have

$$
\begin{aligned}
\left\|\left(Q_{2}^{\prime} x\right)(t+h)-\left(Q_{2}^{\prime} x\right)(t)\right\| \\
\leq \int_{0}^{t+h} \mathcal{T}_{q}(t+h-s) f(s, x(s),(H x)(s)) d s-\int_{0}^{t} \mathcal{T}_{q}(t-s) f(s, x(s),(H x)(s)) d s \\
\leq \int_{0}^{t}\left[\mathcal{T}_{q}(t+h-s)-\mathcal{T}_{q}(t-s)\right] f(s, x(s),(H x)(s)) d s \\
\quad+\int_{t}^{t+h} \mathcal{T}_{q}(t+h-s) f(s, x(s),(H x)(s)) d s \\
\leq \int_{0}^{t}\left[\mathcal{T}_{q}(t+h-s)-\mathcal{T}_{q}(t-s)\right] f(s, x(s),(H x)(s)) d s \\
\quad+M \int_{t}^{t+h}\|f(s, 0,0)\| d s+M h\left(\alpha_{1} r+\alpha_{2} k^{*} r\right) .
\end{aligned}
$$

Since $\mathcal{T}_{q}(t)$ is strongly continuous for $t \geq 0$ and $f$ is compact, it follows that $Q_{2}^{\prime}\left(B_{r}\right) \subset B_{r}$ is equicontinuous, and the set $\left\{\mathcal{T}_{q}(t-s) f(s, x(s),(H x)(s)): s, t \in[0, b], x \in B_{r}\right\}$ is precompact. It is easy to have

$$
Q_{2}^{\prime}\left(B_{r}\right)(t) \subset t \overline{\operatorname{conv}}\left\{\mathcal{T}_{q}(t-s) f(s, x(s),(H x)(s)): s, t \in[0, b], x \in B_{r}\right\}
$$

Thus we have $Q_{2}^{\prime}\left(B_{r}\right)(t) \subset \mathbb{X}$ is precompact. Then $Q^{\prime}=Q_{1}^{\prime}+Q_{2}^{\prime}$ is a condensing operator on $B_{r}$. By Lemma 2.4, $Q^{\prime}$ has a fixed point $x$ on $B_{r}$. It is easy to prove that $x$ is a mild solution of system (1.1) Similar to the proof of Theorem 3.1, it is easy to see that system (1.1) is approximately controllable on $[0, b]$. The proof is completed.

\section{Existence of optimal controls}

In this section, we suppose that $\mathbb{Y}$ is a separable Banach space. $w_{f}(\mathbb{Y})$ represents a class of nonempty, closed, and convex subsets of $\mathbb{Y}$. The multifunction $w: I \rightarrow w_{f}(\mathbb{Y})$ is a measurable and $w(\cdot) \subset E$, where $E$ is a bounded set of $\mathbb{Y}$, the admissible control set 
$U_{a d}=\left\{u \in L^{1}(E) \mid u(t) \in w(t)\right.$ a.e. $\}$. Then $u(t)$ is nonempty. Consider the following fractional control system:

$$
\left\{\begin{array}{c}
{ }^{C} D_{t}^{q} x(t)=A x(t)+f(t, x(t),(H x)(t))+C(t) u(t), \\
t \in I=[0, b], 1<q<2, u \in U_{a d}, \\
x(0)+g_{1}(x)=x_{0} \in \mathbb{X}, \quad x^{\prime}(0)+g_{2}(x)=x_{1} \in \mathbb{X},
\end{array}\right.
$$

where $C \in L^{\infty}(I, L(\mathbb{Y}, \mathbb{X}))$. It is easy to see that $C u \in L^{1}(I, \mathbb{X})$ for all $u \in U_{a d}$.

Let $x^{u}$ be a mild solution of system (4.1) corresponding to a control $u \in U_{a d}$. We consider the Bolza problem (P): find a optimal pair $\left(x^{0}, u^{0}\right) \in C(I, \mathbb{X}) \times U_{a d}$ such that

$$
J\left(x^{0}, u^{0}\right) \leq J\left(x^{u}, u\right), \quad \text { for all } u \in U_{a d},
$$

where

$$
J\left(x^{u}, u\right)=\phi\left(x^{u}(b)\right)+\int_{0}^{b} l\left(t, x^{u}(t), u(t)\right) d t .
$$

Here, we introduce the following assumptions:

(HL) The functional $l: I \times \mathbb{X} \times \mathbb{Y} \rightarrow \mathbb{R} \cup\{\infty\}$ is Borel measurable.

$l(t, \cdot, \cdot)$ is sequentially lower semicontinuous on $\mathbb{X} \times \mathbb{Y}$ for almost all $t \in I$.

$l(t, \cdot, \cdot)$ is convex on $\mathbb{Y}$ for each $x \in \mathbb{X}$ and almost all $t \in I$.

There exist constants $d \geq 0, e>0, \varphi$ is nonnegative, and $\varphi \in L^{1}(I, \mathbb{R})$ such that

$$
l(t, x, u) \geq \varphi(t)+d\|x\|+e\|u\|_{\mathbb{Y}}^{p}
$$

Theorem 4.1 Let $A$ be the infinitesimal generator of an analytic compact semigroup $\{T(t), t \geq 0\}$. In addition to the assumptions of Theorem 3.1, we suppose that condition $(\mathrm{HL})$ holds. Then the Bolza problem $(\mathrm{P})$ admits at least one optimal pair on $C(I, \mathbb{X}) \times U_{a d}$.

Proof If $\inf \left\{J\left(x^{u}, u\right) \mid u \in U_{a d}\right\}=+\infty$, there is nothing to prove. So we assume that $\inf \left\{\left(x^{u}, u\right) \mid u \in U_{a d}\right\}=\eta<+\infty$. Since condition (HL) holds, we have

$$
\begin{aligned}
J\left(x^{u}, u\right) & \geq \phi\left(x^{u}(b)\right)+\int_{0}^{b} \varphi(t) d t+d \int_{0}^{b}\left\|x^{u}(t)\right\| d t+e \int_{0}^{b}\|u(t)\|_{\mathbb{Y}}^{p} d t \\
& \geq-\sigma>-\infty .
\end{aligned}
$$

Here, $\sigma>0$ is a constant, so $\eta \geq-\sigma>-\infty$.

By the definition of infimum there exists a minimizing sequence of feasible pair $\left\{\left(x^{m}, u^{m}\right)\right\} \subset A_{a d}$, where $A_{a d} \equiv\{(x, u) \mid x$ is a mild solution of system (4.1) corresponding to $\left.u \in U_{a d}\right\}$, such that $J\left(x^{n}, u^{n}\right) \rightarrow \eta$ as $n \rightarrow+\infty$. Since $\left\{u^{n}\right\} \subseteq U_{a d}$, $\left\{u^{n}\right\}$ is bounded in $L^{1}(I, \mathbb{Y})$, there exists a subsequence, relabeled as $\left\{u^{n}\right\}$, and $u^{0} \in L^{1}(I, \mathbb{Y})$ such that $u^{n}$ weakly converges to $u^{0}$ in $L^{1}(I, \mathbb{Y})$. Since the admissible control set $U_{a d}$ is convex and closed, we have $u^{0} \in U_{a d}$.

Suppose that $x^{n}$ is a mild solution of system (4.1) corresponding to $u^{n}$, and $x^{n}$ satisfies

$$
\begin{aligned}
x^{n}(t)= & \mathcal{S}_{q}(t)\left(x_{0}-g_{1}\left(x^{n}\right)\right)+\mathcal{K}_{q}(t)\left(x_{1}-g_{2}\left(x^{n}\right)\right)+\int_{0}^{t} \mathcal{T}_{q}(t-s) B u^{n}(s) d s \\
& +\int_{0}^{t} \mathcal{T}_{q}(t-s) f\left(s, x^{n}(s),\left(H x^{n}\right)(s)\right) d s .
\end{aligned}
$$


From $\left(\mathrm{H}_{2}\right)$, we find that $f\left(s, x^{n}(s),\left(H x^{n}\right)(s)\right)$ is a bounded continuous operator from $I$ to $\mathbb{X}$. Because $f\left(s, x^{n}(s),\left(H x^{n}\right)(s)\right) \in L^{1}(I, \mathbb{X})$ is bounded, there exists a subsequence, relabeled as $\left\{f\left(s, x^{n},\left(H x^{n}\right)(s)\right)\right\}$ and $\widehat{f}(s, x,(H x)(s)) \in L^{1}(I, \mathbb{X})$ such that $f\left(s, x^{n},\left(H x^{n}\right)(s)\right)$ weakly converges to $\widehat{f}(s, x,(H x)(s))$.

We denote

$$
\left(Q_{1}^{\prime \prime} x\right)(t)=\int_{0}^{t} \mathcal{T}_{q}(t-s) f(s, x(s),(H x)(s)) d s, \quad\left(Q_{2}^{\prime \prime} x\right)(t)=\int_{0}^{t} \mathcal{T}_{q}(t-s) B u(s) d s .
$$

By $\left(\mathrm{H}_{1}\right)$ and $\left(\mathrm{H}_{2}\right)$, we can see that $\left\|\left(Q_{1}^{\prime \prime} x\right)(t)\right\|$ is bounded. It is not difficult to see that $\left\|\left(Q_{1}^{\prime \prime} x\right)(t)\right\|$ is compact and equicontinuous in $\mathbb{X}$. Then by the Ascoli-Arzela theorem, $\left\{\left(Q_{1}^{\prime \prime} x\right)(t)\right\}$ is relatively compact in $C(I, \mathbb{X})$. Since $Q_{1}^{\prime \prime}$ is linear and continuous, $Q_{1}^{\prime \prime}$ is a strongly continuous operator. Thus we see that $Q_{1}^{\prime \prime} x^{n}$ strongly converges to $Q_{1}^{\prime \prime} x$ in $C(I, \mathbb{X})$. Similarly, we can conclude that $Q_{2}^{\prime \prime}$ is a strongly continuous operator. Next, we consider the following controlled system:

$$
\left\{\begin{array}{l}
{ }^{C} D_{t}^{q} x(t)=A x(t)+\widehat{f}(t, x(t),(H x)(t))+C(t) u^{0}(t), \quad t \in[0, b], 1<q<2, u \in U_{a d}, \\
x(0)+g_{1}(x)=x_{0} \in \mathbb{X}, \quad x^{\prime}(0)+g_{2}(x)=x_{1} \in \mathbb{X} .
\end{array}\right.
$$

Similar to Theorem 3.1, it is easy to prove that system (4.8) has a mild solution,

$$
\begin{aligned}
\widehat{x}(t)= & \mathcal{S}_{q}(t)\left(x_{0}-g_{1}(\widehat{x})\right)+\mathcal{K}_{q}(t)\left(x_{1}-g_{2}(\widehat{x})\right)+\int_{0}^{t} \mathcal{T}_{q}(t-s) C(s) u^{0}(s) d s \\
& +\int_{0}^{t} \mathcal{T}_{q}(t-s) \widehat{f}(s, x(s),(H x)(s)) d s .
\end{aligned}
$$

For each $t \in I, x^{n}(\cdot), \widehat{x}(\cdot) \in \mathbb{X}$, we get

$$
\begin{aligned}
\left\|x^{n}(t)-\widehat{x}(t)\right\| \leq & \mathcal{S}_{q}(t)\left\|g_{1}\left(x^{n}\right)-g_{1}(\widehat{x})\right\|+\mathcal{K}_{q}(t)\left\|g_{2}\left(x^{n}\right)-g_{2}(\widehat{x})\right\| \\
& +\int_{0}^{t} \mathcal{T}_{q}(t-s) C(s)\left(u^{n}(s)-u^{0}(s)\right) d s \quad \text { denoted by } P_{1} \\
& +\int_{0}^{t} \mathcal{T}_{q}(t-s)\left(f\left(s, x^{n}(s),\left(H x^{n}\right)(s)\right)-\widehat{f}(s, x(s),(H x)(s))\right) d s \\
& \text { denoted by } P_{2},
\end{aligned}
$$

which implies that

$$
\left\|x^{n}-\widehat{x}\right\| \leq \frac{P_{1}+P_{2}}{1-M\left(\beta_{1}+\beta_{2}\right)} .
$$

Therefore we can infer that $x^{n}$ strongly converges to $\widehat{x}$ in $C(I, \mathbb{X})$ as $n \rightarrow \infty$.

From $\left(\mathrm{H}_{2}\right)-\left(\mathrm{H}_{4}\right), f\left(s, x^{n}(s),\left(H x^{n}\right)(s)\right)$ strongly converges to $f(s, \widehat{x}(s),(H \widehat{x})(s))$ in $C(I, \mathbb{X})$ as $n \rightarrow \infty$. From the uniqueness of the limit, we obtain $\widehat{f}(s, x(s),(H x)(s))=f(s, \widehat{x}(s),(H \widehat{x})(s))$. Then

$$
\begin{aligned}
\widehat{x}(t)= & \mathcal{S}_{q}(t)\left(x_{0}-g_{1}(\widehat{x})\right)+\mathcal{K}_{q}(t)\left(x_{1}-g_{2}(\widehat{x})\right)+\int_{0}^{t} \mathcal{T}_{q}(t-s) C(s) u^{0}(s) d s \\
& +\int_{0}^{t} \mathcal{T}_{q}(t-s) f(s, \widehat{x}(s),(H \widehat{x})(s)) d s .
\end{aligned}
$$


From assumption (HL) and Balder's theorem, we can infer that

$$
\begin{aligned}
\eta & =\phi\left(x^{n}(b)\right)+\lim _{n \rightarrow \infty}+\int_{0}^{b} l\left(t, x^{n}(t), u^{n}(t)\right) d t \\
& \geq \phi(\widehat{x}(b))+\int_{0}^{b} l\left(t, \widehat{x}, u^{0}(t)\right) d t=J\left(\widehat{x}, u^{0}\right) \geq \eta,
\end{aligned}
$$

which implies that $J$ attains its minimum at $\left(\widehat{x}, u^{0}\right) \in C(I, \mathbb{X}) \times U_{a d}$. The proof is completed.

\section{Applications}

Example 5.1 Consider optimal controls for fractional control system of order $q=\frac{3}{2}$ as follows:

$$
\left\{\begin{aligned}
& \frac{\partial^{\frac{3}{2}}}{\partial t^{\frac{3}{2}}} x(t, y)= \frac{\partial^{2}}{\partial y^{2}} x(t, y)+\frac{e^{-t}}{e^{t}+e^{-t}} \cos \left[x(t, y)+\int_{0}^{1} x(s, y) d s+\int_{0}^{t} h(t, s) x(s, y) d s\right] \\
&+\int_{0}^{1} k(t, s) u(s, y) d s, \quad y \in[0,1], t \in[0,1], u \in U_{a d} \\
& x(t, 0)=x(t, 1)=0, \quad t>0 \\
& x(0, y)=\sum_{i=0}^{\sigma_{1}} \int_{0}^{1} k_{1}(t, s) x\left(s_{i}, y\right) d y+\sum_{i=0}^{\sigma_{1}} \int_{0}^{1} k_{2}(t, s) \frac{\partial}{\partial y} x\left(s_{i}, y\right) d y \\
& x^{\prime}(0, y)=\sum_{i=0}^{\sigma_{2}} \int_{0}^{1} k_{1}(t, s) x\left(s_{i}, y\right) d y+\sum_{i=0}^{\sigma_{2}} \int_{0}^{1} k_{2}(t, s) \frac{\partial}{\partial y} x\left(s_{i}, y\right) d y
\end{aligned}\right.
$$

with a cost function

$$
J(x, u)=\int_{0}^{1} \int_{0}^{1}|x(t, y)|^{2} d y d t+\int_{0}^{1} \int_{0}^{1}|u(t, y)|^{2} d y d t+\int_{0}^{1}|x(b, y)|^{2} d y,
$$

where $\sigma_{i} \in \mathbb{N}, 0<s_{0}<s_{1}<\cdots<s_{\sigma_{i}}<1, h, k \in C\left([0,1] \times[0,1], \mathbb{R}^{+}\right), k_{i} \in L^{2}\left([0,1] \times[0,1], \mathbb{R}^{+}\right)$, $i=1,2$.

As a similar method to the example of the example in [26], let $\mathbb{X}=\mathbb{Y}=\left(L^{2}([0,1]),\|\cdot\|_{2}\right)$. Operator $A: D(A) \rightarrow \mathbb{X}$ is defined by $D(A)=\left\{x \in \mathbb{X} \mid x^{\prime}, x^{\prime \prime} \in \mathbb{X}, x(0)=x(1)=0\right\}$ with $A x=$ $-x^{\prime \prime}$, then $A$ generates a compact, analytic semigroup $T(\cdot)$ of uniformly bounded linear operator, and assumption $\left(\mathrm{H}_{1}\right)$ is satisfied. Moreover, the eigenvalues of $A$ are $n^{2} \pi^{2}$ and the corresponding normalized eigenvectors are $e_{n}(u)=\sqrt{2} \sin (n \pi u), n=1,2, \ldots$.

Here, we take the control function $u: T x([0,1]) \rightarrow \mathbb{R}$ such that $u \in L^{2}(T x([0,1]))$. Then $t \rightarrow u(t, \cdot)$ going from $[0,1]$ into $\mathbb{Y}$ is measurable. Set $U(t)=\left\{u \in \mathbb{Y} \mid\|u\|_{\mathbb{Y}} \leq \vartheta\right\}$ where $\vartheta \in L^{2}\left(I, \mathbb{R}^{+}\right)$. We also restrict the admissible controls $U_{a d}$ to be all the $u \in L^{2}(T x([0,1]))$ such that $\|u(t, \cdot)\|_{2} \leq \vartheta(t)$, a.e.

Let us denote $C(I, \mathbb{X})$ a Banach space equipped with supnorm $\|\cdot\|$. Let $x(t)(y)=x(t, y)$, $(H x)(t)(y)=\left(\int_{0}^{t} h(t, s) x(s) d s\right)(y), C(t) u(t)(y)=\left(\int_{0}^{1} k(t, s) u(s) d s\right)(y)$. Define $f:[0,1] \times \mathbb{X} \times$ $\mathbb{X} \rightarrow \mathbb{X}$ by

$$
f(t, x(t),(H x)(t))(y)=\frac{e^{-t}}{e^{t}+e^{-t}} \cos \left(x(t)+\int_{0}^{1} x(s) d s+\int_{0}^{t} h(t, s) x(s) d s\right)(y)
$$

and $g_{1}, g_{2}: C(I, \mathbb{X}) \rightarrow \mathbb{X}$ by

$$
g_{1}(x)(y)=\left(\sum_{i=0}^{\sigma_{1}}(K x)\left(t_{i}\right)\right)(y) \quad \text { for } x \in C(I, \mathbb{X}),
$$




$$
g_{2}(x)(y)=\left(\sum_{i=0}^{\sigma_{2}}(K x)\left(t_{i}\right)\right)(y) \quad \text { for } x \in C(I, \mathbb{X})
$$

where $K: \mathbb{X} \rightarrow \mathbb{X}$ are defined by

$$
(K x)(s)=\int_{0}^{1} k_{1}(t, s) x(s) d s+\int_{0}^{1} k_{2}(t, s) x^{\prime}(s) d s, \quad \text { for all } x \in \mathbb{X} .
$$

It is easy to see that

$$
(K(x-y))(s)=\int_{0}^{1} k_{1}(t, s)(x-y)(s) d s+\int_{0}^{1} k_{2}(t, s)\left(x^{\prime}-y^{\prime}\right)(s) d s, \quad \text { for all } x \in \mathbb{X} .
$$

The system (5.1) can be transformed into the following type:

$$
\left\{\begin{array}{l}
{ }^{C} D_{t}^{q} x(t)=A x(t)+f(t, x(t),(H x)(t))+C(t) u(t), \quad t \in I, u \in U_{a d}, \\
x(0)+g_{1}(x)=x_{0} \in \mathbb{X}, \quad x^{\prime}(0)+g_{2}(x)=x_{1} \in \mathbb{X},
\end{array}\right.
$$

with a cost function

$$
J(x, u)=\|x(b)\|+\int_{0}^{b}\left(\|x(t)\|^{2}+\|u(t)\|_{\mathbb{Y}}^{2}\right) d t,
$$

we can verify $(\mathrm{HL})$ is satisfied. It is also not difficult to know

$$
\|f(t, x(t),(H x)(t))\| \leq \frac{e^{-t}}{e^{t}+e^{-t}}=\varphi(t), \quad \varphi(t) \in L^{\infty}\left(I, \mathbb{R}^{+}\right) .
$$

Then there exist $\mu_{1}(t)=\varphi(t), \mu_{2}(t)=\mu_{3}(t) \equiv 0$ such that condition $\left(\mathrm{H}_{2}\right)$ holds.

Meanwhile, one finds from example in [25] that $g_{1}$ and $g_{2}$ are completely continuous operators from $C(I, \mathbb{X})$ to $\mathbb{X}$ and satisfy

$$
\begin{aligned}
& \left\|g_{1}(x)-g_{1}(y)\right\| \leq \sigma_{1}\left(c_{12}+c_{22}\right)\|x-y\|, \\
& \left\|g_{2}(x)-g_{2}(y)\right\| \leq \sigma_{2}\left(c_{12}+c_{22}\right)\|x-y\|,
\end{aligned}
$$

where the definitions of constants $c_{12}$ and $c_{22}$ are the same as that in [26]. Let $\beta_{1}=\sigma_{1}\left(c_{12}+\right.$ $\left.c_{22}\right)$ and $\beta_{2}=\sigma_{2}\left(c_{12}+c_{22}\right)$, it is easy to verify that $\left(\mathrm{H}_{3}\right)$ holds. Since the operator $H$ in the nonlinear term $f$ is linear, condition $\left(\mathrm{H}_{4}\right)$ is satisfied automatically. By Theorem 4.1, we can conclude that the system (5.1) has at least one optimal pair, while the condition $M\left(\sigma_{1}+\sigma_{2}\right)\left(c_{12}+c_{22}\right)<1$ holds.

Remark 5.1 In order to describe various problems in nature and science which undergo abrupt changes at certain instants during the evolution process, impulsive fractional differential equations are emerging as an important class of system models. Among the previous and known results, few people have discussed the control problems for impulsive differential equations of order $1<q<2$. Using the same methods and ideas in this paper, one can obtain the approximate controllability and optimal controls results of fractional impulsive differential equations with nonlocal conditions of order $1<q<2$. 


\section{Competing interests}

The authors declare that they have no competing interests.

\section{Authors' contributions}

The authors have contributed to this work on an equal basis. All authors read and approved the final manuscript.

\section{Author details}

${ }^{1}$ Department of Automation, China University of Petroleum (Beijing), Beijing, 102249, China. ${ }^{2}$ School of Mathematical Sciences, Qufu Normal University, Qufu, Shandong 273165, China.

\section{Acknowledgements}

The fourth author was supported financially by the National Natural Science Foundation of China (11371221), the Specialized Research Foundation for the Doctoral Program of Higher Education of China (20123705110001), and the Program for Scientific Research Innovation Team in Colleges and Universities of Shandong Province.

Received: 26 July 2014 Accepted: 2 February 2015 Published online: 04 March 2015

\section{References}

1. Hernández, E, O'Regan, D, Balachandran, K: On recent developments in the theory of abstract differential equations with fractional derivatives. Nonlinear Anal. 73, 3462-3471 (2010)

2. Zhou, Y, Jiao, F: Existence of mild solutions for fractional neutral evolution equations. Comput. Math. Appl. 59, 1063-1077 (2010)

3. Shu, $X$, Lai, Y, Chen, Y: The existence of mild solutions for impulsive fractional partial differential equations. Nonlinear Anal. 74, 2003-2011 (2011)

4. Zhang, X, Huang, X, Liu, Z: The existence and uniqueness of mild solutions for impulsive fractional equations with nonlocal conditions and infinite delay. Nonlinear Anal. Hybrid Syst. 4, 775-781 (2010)

5. Rashid, MHM, Al-Omari, A: Local and global existence of mild solutions for impulsive fractional semilinear integro-differential equation. Commun. Nonlinear Sci. Numer. Simul. 16, 3493-3503 (2011)

6. Mophou, GM: Existence and uniqueness of mild solutions to impulsive fractional differential equations. Nonlinear Anal. 72, 1604-1615 (2010)

7. Shu, X, Wang, Q: The existence and uniqueness of mild solutions for fractional differential equations with nonlocal conditions of order $1<\alpha<2$. Comput. Math. Appl. 64, 2100-2110 (2012)

8. Balachandran, K, Kiruthika, S, Trujillo, JJ: Existence results for fractional impulsive integrodifferential equations in Banach spaces. Commun. Nonlinear Sci. Numer. Simul. 16, 1970-1977 (2011)

9. Li, $\mathrm{K}, \mathrm{Jia}, \mathrm{J}$ : Existence and uniqueness of mild solutions for abstract delay fractional differential equations. Comput. Math. Appl. 62, 1398-1404 (2011)

10. Hernández, E, O'Regan, D, Balachandran, K: Existence results for abstract fractional differential equations with nonlocal conditions via resolvent operators. Indag. Math. 24,68-82 (2013)

11. Kavitha, $V$, Wang, P, Murugesu, R: Existence of weighted pseudo almost automorphic mild solutions to fractional integro-differential equations. J. Fract. Calc. Appl. 4, 37-55 (2013)

12. Dabas, J, Chauhan, A: Existence and uniqueness of mild solution for an impulsive neutral fractional integro-differential equation with infinite delay. Math. Comput. Model. 57, 754-763 (2013)

13. Olszowy, L: Existence of mild solutions for the semilinear nonlocal problem in Banach spaces. Nonlinear Anal. 81, 211-223 (2013)

14. Sakthivel, R, Ren, Y: Approximate controllability of fractional differential equations with state dependent delay. Results Math. 63, 949-963 (2013)

15. Li, K, Peng, J, Gao, J: Controllability of nonlocal fractional differential systems of order $\alpha \in(1,2]$ in Banach spaces. Rep. Math. Phys. 71(1), 33-43 (2013)

16. Sakthivel, R, Ganesh, R, Ren, Y, Anthoni, SM: Approximate controllability of nonlinear fractional dynamical systems. Commun. Nonlinear Sci. Numer. Simul. 18, 3498-3508 (2013)

17. Sakthivel, R, Ren, Y, Mahmudov, NI: On the approximate controllability of semilinear fractional differential systems Comput. Math. Appl. 62, 1451-1459 (2011)

18. Sakthivel, R, Ganesh, R, Suganya, S: Approximate controllability of fractional neutral stochastic system with infinite delay. Rep. Math. Phys. 70, 291-311 (2012)

19. Sakthivel, R, Ganesh, R, Anthoni, SM: Approximate controllability of fractional nonlinear differential inclusions. Appl. Math. Comput. 225, 708-717 (2013)

20. Fečkan, M, Wang, JR, Zhou, Y: Controllability of fractional functional evolution equations of Sobolev type via characteristic solution operators. J. Optim. Theory Appl. 156, 79-95 (2013)

21. Ganesh, R, Sakthivel, R, Mahmudov, NI, Anthoni, SM: Approximate controllability of fractional integrodifferential evolution equations. J. Appl. Math. 2013, Article ID 291816 (2013)

22. Ravichandran, C, Trujillo, J: Controllability of impulsive fractional functional integrodifferential equations in Banach spaces. J. Funct. Spaces Appl. 2013, Article ID 812501 (2013)

23. Balachandran, K, Govindaraj, V, Rodríguez-Germa, L, Trujillo, JJ: Controllability results for nonlinear fractional-order dynamical systems. J. Optim. Theory Appl. 156, 33-44 (2013)

24. Liu, Z, Li, X: On the controllability of impulsive fractional evolution inclusions in Banach spaces. J. Optim. Theory Appl. 156, 167-182 (2013)

25. Wang, JR, Zhou, Y, Medved', M: On the solvability and optimal controls of fractional integrodifferential evolution systems with infinite delay. J. Optim. Theory Appl. 152, 31-50 (2012)

26. Wang, JR, Zhou, Y, Wei, W, Xu, H: Nonlocal problems for fractional integrodifferential equations via fractional operators and optimal controls. Comput. Math. Appl. 62, 1427-1441 (2011)

27. Liu, X, Liu, Z, Bin, M: The solvability and optimal controls for some fractional impulsive equations of order $1<\alpha<2$. Abstr. Appl. Anal. 2014, Article ID 142067 (2014) 
28. Guatteri, G, Masiero, F: On the existence of optimal controls for SPDES with boundary noise and boundary control. SIAM J. Control Optim. 51(3), 1909-1939 (2013)

29. Liu, X, Liu, Z, Han, J: The solvability and optimal controls for some fractional impulsive equation. Abstr. Appl. Anal. 2013, Article ID 914592 (2013)

30. Debbouche, A, Baleanu, D: Controllability of fractional evolution nonlocal impulsive quasilinear delay integro-differential systems. Comput. Math. Appl. 62, 1442-1450 (2011)

31. Debbouche, A, Baleanu, D: Exact null controllability for fractional nonlocal integrodifferential equations via implicit evolution system. J. Appl. Math. 2012, Article ID 931975 (2012)

32. Debbouche, A, Torres, DFM: Approximate controllability of fractional nonlocal delay semilinear systems in Hilbert spaces. Int. J. Control 86(9), 1577-1585 (2013)

33. Debbouche, A, Torres, DFM: Approximate controllability of fractional delay dynamic inclusion with nonlocal control conditions. Appl. Math. Comput. 243, 161-175 (2014)

34. Debbouche, A, Nieto, Jj: Sobolev type fractional abstract evolution equations with nonlocal conditions and optimal multi-controls. Appl. Math. Comput. 245, 74-85 (2014)

35. Debbouche, A, Torres, DFM: Sobolev type fractional dynamic equations and optimal multi-integral controls with fractional nonlocal conditions. Fract. Calc. Appl. Anal. arXiv:1409.6028v1

\section{Submit your manuscript to a SpringerOpen ${ }^{\mathcal{O}}$ journal and benefit from:}

- Convenient online submission

Rigorous peer review

- Immediate publication on acceptance

Open access: articles freely available online

- High visibility within the field

- Retaining the copyright to your article 